\title{
Short-Period Variables in ASAS Photometry
}

\author{
J. Molenda-Żakowicz ${ }^{1}$ \\ ${ }^{1}$ Institute of Astronomy, Kopernika 11, 51-622 Wrocław
}

\begin{abstract}
We present results of our study of short-period variables in ASAS photometry. We focus on slowly pulsating B stars (SPBs), most of which were discovered by the Hipparcos satellite. We discuss the problem of aliasing in the ASAS photometry and its influence on the identification of frequencies of SPBs. Finally, we show that the ASAS photometry can be used not only to confirm the Hipparcos frequencies and amplitudes but it can also give new results for selected SPBs.
\end{abstract}

\section{Introduction}

The ASAS (All Sky Automatic Survey) project aims for monitoring variability of all objects in the sky brighter that 14 mag. Its prototype instrument and data pipeline were developed by Dr. Pojmański (Pojmański 1997). In 1997-2000 ASAS observed in the I-band (ASAS2 project) and since 2001 in $V$ (ASAS-3 project). It succeeded in discovering more than 5500 new variables (Pojmański 2000 and 2002) and was used for studying Cepheids in the Magellanic Clouds (Pietrukowicz 2001, 2002) and the Galaxy (Beltrame \& Poretti 2002).

In this work, we analyze ASAS-3 photometry of stars classified as SPB in the Hipparcos Catalogue (ESA 1997). We discuss ASAS observing and spectral window and present results obtained for selected stars.

\section{Observing and Spectral Windows}

In the ASAS-3 observing schedule each of the preselected fields is observed once per night. However, the fields overlap so that many stars are observed several times per night. We show a typical ASAS observing window in the left panel of Fig. 2. This window (compiled for HD 52057 from three separate data sets) covers a time span of 2.3 years and consists of 152 observations indicated by impulses. In this figure, Observations are gathered in three clumps inside which sampling is close to one per night. Observations sampled more frequently are rare but also present.

Such sampling produces significant daily aliases in the spectral window; in the left panel of Fig. 1 we show an example - the spectral window computed for IS Lup. Windows of this shape, with daily aliases getting lower for higher frequencies, can be computed for $\sim 50 \%$ stars observed by ASAS. The other stars have spectral windows with a $2 \mathrm{c} / \mathrm{d}$ peak higher than the one at $1 \mathrm{c} / \mathrm{d}$. We find such spectral windows for stars which were observed at the beginning and then at the end of the majority of observing nights. As an example, we show the spectral window for HD 52057 in the middle panel of Fig. 1 . For few stars we find spectral windows with the $3 \mathrm{c} / \mathrm{d}$ peak higher than the 1 and $2 \mathrm{c} / \mathrm{d}$ ones. Windows of this shape occur for stars which are observed sparsely and for which observations are clumped in 


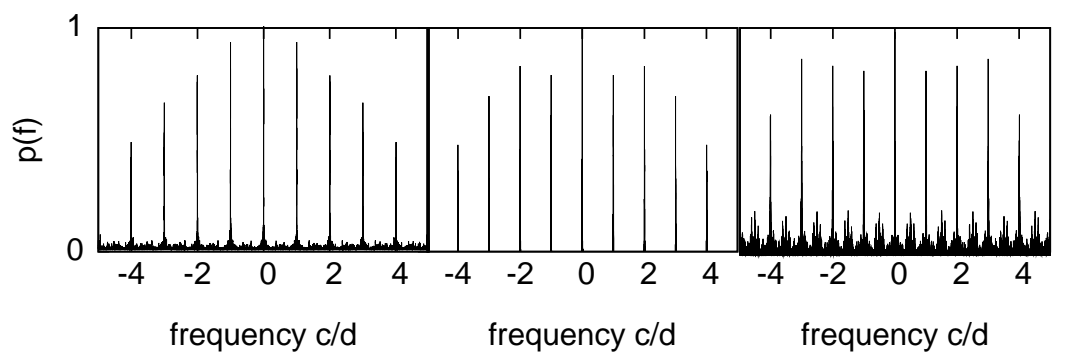

Figure 1: Spectral windows of ASAS photometry. left: IS Lup, middle: HD 52057, right: V 1370 Ori.

Table 1: Number of observations, frequencies and amplitudes detected in ASAS photometry for 17 stars classified as SPB in the Hipparcos Catalogue. Asterisks indicate that the highest peak in periodogram indicated is an alias of the correct frequency.

\begin{tabular}{|c|c|c|c|c|c|c|c|}
\hline Name & $N_{\text {obs }}$ & $\begin{array}{c}\mathrm{f} \\
(\mathrm{c} / \mathrm{d})\end{array}$ & $\underset{(\mathrm{mmag})}{\mathrm{A}}$ & Name & $N_{\text {obs }}$ & $\begin{array}{c}\mathrm{f} \\
(\mathrm{c} / \mathrm{d})\end{array}$ & $\begin{array}{c}\mathrm{A} \\
(\mathrm{mmag})\end{array}$ \\
\hline CGHyi & 123 & $\begin{array}{c}1.33482^{*} \\
1.44480\end{array}$ & $\begin{array}{c}18.19 \\
9.66\end{array}$ & V386 Pup & 39 & $1.21260^{*}$ & 64.66 \\
\hline V1370 Ori & 45 & $1.11300 *$ & 22.98 & MY Vel & 100 & 0.80769 & 25.43 \\
\hline V726 Mon & 65 & $2.32456 *$ & 23.04 & V363 Vel & 119 & 0.40717 & 26.04 \\
\hline NZ CMa & 117 & $0.88972 *$ & 11.68 & PR Vel & 116 & 0.80727 & 18.25 \\
\hline $\mathrm{KP} \mathrm{CMa}$ & 106 & 0.93566 & 22.02 & & & 0.87613 & 11.99 \\
\hline LM CMa & 121 & 0.43212 & 24.03 & PZ Vel & 158 & 1.17949 & 17.05 \\
\hline \multirow[t]{2}{*}{ HD 52057} & 152 & 0.98423 & 14.25 & LY Vel & 147 & 1.34908 & 10.18 \\
\hline & & 0.79958 & 12.37 & V483 Car & 151 & 1.22191 & 15.46 \\
\hline V375 Pup & 122 & 0.58761 & 24.29 & IS Lup & 185 & 1.16974 & 16.42 \\
\hline $\mathrm{LZ} \operatorname{Tr} \mathrm{A}$ & 193 & $1.75355 *$ & 20.82 & & & & \\
\hline
\end{tabular}

very narrow time spans at the beginning and at the end of observing nights. An example is the spectral window of V 1370 Ori shown in the right panel of Fig. 1.

\section{Results for SPBs}

We analyzed ASAS photometry of 72 stars classified as SPB in the Hipparcos Catalogue. We downloaded observations of southern SPBs form the ASAS home page (http://www. astrouw.edu.pl/ gp/asas/asas.html) while for the northern ones we used data kindly provided by Dr. Pojmański. In case of 55 stars we could not perform analysis of variability. Most of these stars are too bright for ASAS and their magnitudes are saturated, show high scatter and significant trends. We did not analyze stars with a number of observations less than $\sim 40$. Eventually, we were left with 17 stars in whose photometry we sought periodic variability. We list the stars in Table 1 . In the first column we give the names or HD numbers of the stars, then we list the number of observations used for analysis, the values of detected frequencies and fitted amplitudes. Frequencies of six stars are indicated with an asterisk. We indicated in this way stars, for which the highest peak in periodogram occurred at an alias of the correct frequency. (We identified correct frequencies using Hipparcos photometry which does not show daily aliases.) As we found, the problem of aliasing is most severe for stars with a small number of observations (V 1370 Ori, V 726 Mon and V 386 Pup) or/and a spectral window with the highest peak at 2 (CG Cyg) or $3 \mathrm{c} / \mathrm{d}$ (V 1370 Ori).

The ASAS photometry reproduces quite well also amplitudes known from Hipparcos pho- 


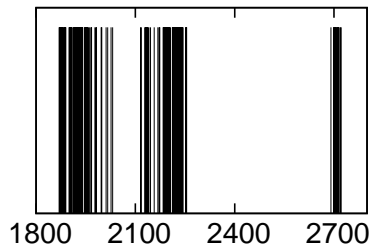

HJD-2450000
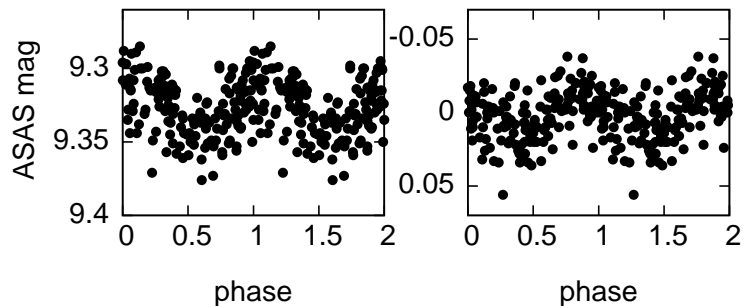

Figure 2: HD 52057: left: observing window, middle: ASAS magnitudes phased with frequency $f_{1}=$ $0.98423 \mathrm{c} / \mathrm{d}$, right residuals of ASAS magnitudes phased with frequency $f_{2}=0.79958 \mathrm{c} / \mathrm{d}$.

tometry. However, due to the scatter in the two photometric data sets and because of the probability of imperfect fits of amplitudes, a detailed comparison can not be performed.

The frequencies present in the ASAS photometry agree with the Hipparcos ones much better. We confirmed frequencies known from Hipparcos photometry for 11 monoperiodic and two doubly-periodic stars, namely, CG Hyi and PR Vel. For three other doubly-periodic Hipparcos SPBs, namely KP CMa, PZ Vel and V 483 Car, we confirm only the first frequencies.

For one star, HD 52057, we detected two frequencies, $f_{1}=0.98423$ and $f_{2}=0.79958 \mathrm{c} / \mathrm{d}$, none of which is present in the Hipparcos photometry. We show the phase diagrams constructed for the two frequencies in Fig. 2. In the Hipparcos Catalogue this star is not classified as variable but Waelkens et al. (1998) find in its $H p$ magnitudes one frequency, $f=0.90872$ $\mathrm{c} / \mathrm{d}$ and classify it as SPB. We show that this star is in fact a doubly-periodic variable with both frequencies typical for SPBs.

Acknowledgments. We are indebted to Dr. Pojmański for providing ASAS observations of the northern SPBs. This work was supported by a KBN grant 5 P03D 01420.

\section{References}

Beltrame, M., Poretti, E. 2002, A\&A 386, L9

ESA 1997, The Hipparcos and Tycho Catalogues, ESA SP-1200, NOORDWIJK, ESA

Pietrukowicz, P. 2001, Acta Astron. 51, 247

Pietrukowicz, P. 2002, Acta Astron. 52, 177

Pojmański, G. 1997, Acta Astron. 47, 467

Pojmański, G. 2000, Acta Astron. 50, 177

Pojmański, G. 2002, Acta Astron. 52, 397

Waelkens, C., Aerts, C., Kestens, E., Grenon, M., Eyer, L. 1998, A\&A 330, 215 